SUPPORTING INFORMATION

for a paper in JACS

Oct. 17, 2019

\title{
Multistructural Anharmonicity Controls the Radical Generation Process in Biofuel Combustion
}

Lili Xing, ${ }^{\dagger}$, Zhandong Wang, ${ }^{\S}$ and Donald G.Truhlar ${ }^{\ddagger}$

"Energy and Power Engineering Institute, Henan University of Science and Technology, Luoyang, Henan 471003, China

\$National Synchrotron Radiation Laboratory, University of Science and Technology of China, Hefei, Anhui 230029, PR China

‡Department of Chemistry, Chemical Theory Center, and Minnesota Supercomputing Institute, University of Minnesota, Minnesota 55455-0431, USA

TABLE OF CONTENTS

Technical details of the computations

Table S1. Mean unsigned errors

Table S2. Cartesian coordinates and absolute energies

Table S3. Conformational-vibrational rotational partition functions of the reactant (R) and transition states (TS1-TS5) of the five reactions

Table S4. Ratios of multistructural torsional anharmonicity factors $\mathrm{F}_{\mathrm{act}}^{\mathrm{MS}-\mathrm{T}}$ for each reaction and analogous ratios computed using MS-LQH

Table S5. This table is the same as Table 5 except that it has more temperatures.

Table S6. Ratios of MS-T product ratios to SS-QH product ratios

S-14

Table S7. Calculated rate constants using MP-CVT/SCT

S-15

Table S8. This table is the same as Table 8 except that it has another temperature and more significant figures

Figure S1. $F_{X}^{\mathrm{MS}-\mathrm{T}}$ for each species $X(X=\mathrm{R}, \mathrm{TS} 1, \mathrm{TS} 2, \mathrm{TS} 3, \mathrm{TS} 4, \mathrm{TS} 5) \quad \mathrm{S}-17$

Figure S2. Local periodicities

Figure S3. Percentage contributions of selected structures to the transition state partition function

Figure S4. Branching fractions of R1-R5 as functions of temperature 


\section{Technical details of the calculations}

All the electronic structure calculations were performed using Gaussian 16 software package. ${ }^{34}$ The CCSD(T)-F12a calculations were carried out by using the Molpro program. ${ }^{35}$

Both the SCF calculations and the geometry optimizations were performed with tight convergence criteria. The density functional integrations were carried out by a grid of 99 radial shells per atom with 974 angular points per shell.

The dynamics calculations were performed using the Polyrate 2016-2A $A^{39}$ and Gaussrate $2017^{40}$ codes. The step size for the calculation of the MEP was $0.0026 \AA$, and the Hessians were updated every 10 steps along the reaction path. 
Table S1. Mean unsigned errors (MUEs) of 44 model chemistries relative to the CCSD(T)-F12a/jun-cc-pVTZ results. The MUE is averaged over 15 quantitites: $V^{*}, V_{\text {rev }}^{\ddagger}$, and $\Delta E$ for R1-R5.

\begin{tabular}{lc}
\hline & $\begin{array}{c}\text { MUE } \\
\text { (kcal/mol) }\end{array}$ \\
CCSD(T)-F12a/jun-cc-pVTZ & 0.00 \\
M05-2X/MG3S & 0.45 \\
M08-HX/def2-TZVP & 0.46 \\
M06-2X/MG3S & 0.50 \\
M06-2X/jun-cc-pVTZ & 0.55 \\
M05-2X/ def2-TZVP & 0.55 \\
M08-SO/def2-TZVP & 0.57 \\
M08-HX/MG3S & 0.60 \\
M05-2X/jun-cc-pVTZ & 0.60 \\
M05-2X/jul-cc-pVTZ & 0.64 \\
M08-HX/jul-cc-pVTZ & 0.70 \\
M06-2X/jul-cc-pVTZ & 0.76 \\
M06-2X/def2-TZVP & 0.81 \\
MN12-SX/jul-cc-pVTZ & 0.82 \\
MN12-SX/def2-TZVP & 0.86 \\
M08-HX/jun-cc-pVTZ & 0.91 \\
MN15-L/jul-cc-pVTZ & 0.91 \\
MN15/def2-TZVP & 0.96 \\
MN15-L/jun-cc-pVTZ & 0.98 \\
MN12-L/MG3S & 0.98 \\
MN15-L/def2-TZVP & 1.01 \\
MN15-L/MG3S & 1.02 \\
MN15/jun-cc-pVTZ & 1.04 \\
M08-SO/MG3S & 1.11 \\
MN12-L/jul-cc-pVTZ & 1.12 \\
M08-SO/jul-cc-pVTZ & 1.15 \\
MN15/MG3S & \\
MN12-SX/MG3S & 1.16 \\
MN12-SX/jun-cc-pVTZ & \\
\hline & \\
\hline
\end{tabular}




\begin{tabular}{|c|c|}
\hline MPW1K/jul-cc-pVTZ & 1.27 \\
\hline MN12-L/jun-cc-pVTZ & 1.28 \\
\hline MPW1K/jun-cc-pVTZ & 1.37 \\
\hline MN15/jul-cc-pVTZ & 1.39 \\
\hline MN12-L/def2-TZVP & 1.59 \\
\hline MPW1K/def2-TZVP & 1.63 \\
\hline$\omega B 97 X-D / j u n-c c-p V T Z$ & 1.73 \\
\hline M08-SO/jun-cc-pVTZ & 1.76 \\
\hline B3LYP/jun-cc-pVTZ & 1.80 \\
\hline B3LYP/MG3S & 1.99 \\
\hline$\omega B 97 X-D / M G 3 S$ & 2.01 \\
\hline$\omega B 97 X-D / j u l-c c-p V T Z$ & 2.36 \\
\hline B3LYP/jul-cc-pVTZ & 2.41 \\
\hline B3LYP /def2-TZVP & 2.44 \\
\hline$\omega B 97 X-D /$ def2-TZVP & 2.45 \\
\hline MPW1K/MG3S & 2.72 \\
\hline
\end{tabular}


Table S2. Cartesian coordinates (in $\AA$ ) and absolute energies (in hartrees) of M05-2X/MG3S at consistently optimized geometries

$\begin{array}{lrrr}\text { IP } & \text { Absolute energy: }-273.0322776 \text { hartrees } \\ \mathrm{C} & 2.14153200 & -0.71102800 & -0.26237400 \\ \mathrm{H} & 2.52240000 & -0.74939000 & 0.75831600 \\ \mathrm{H} & 2.04401900 & -1.73285100 & -0.62319200 \\ \mathrm{H} & 2.88085300 & -0.20195400 & -0.87657300 \\ \mathrm{C} & 0.80228500 & 0.01701900 & -0.29926000 \\ \mathrm{H} & 0.45288500 & 0.05756700 & -1.32928400 \\ \mathrm{C} & -0.22788100 & -0.76892700 & 0.51129800 \\ \mathrm{H} & -0.31965100 & -1.77088200 & 0.09264200 \\ \mathrm{H} & 0.13117200 & -0.87756100 & 1.53717400 \\ \mathrm{C} & -1.60256000 & -0.13745000 & 0.55479600 \\ \mathrm{H} & -1.56417700 & 0.82189700 & 1.07199800 \\ \mathrm{H} & -2.28755100 & -0.78862200 & 1.09888700 \\ \mathrm{C} & 0.95989700 & 1.44483500 & 0.21206500 \\ \mathrm{H} & 0.05100300 & 2.02399000 & 0.07288100 \\ \mathrm{H} & 1.20900300 & 1.44379500 & 1.27389700 \\ \mathrm{H} & 1.76217300 & 1.95320600 & -0.31817400 \\ \mathrm{O} & -2.04909300 & 0.04213400 & -0.78478100 \\ \mathrm{H} & -2.92902600 & 0.41703300 & -0.77947700\end{array}$

P1 Absolute energy: -272.3563434 s

$\begin{array}{lrrr}\mathrm{C} & -2.23230100 & -0.46842300 & -0.14030600 \\ \mathrm{H} & -2.28614700 & -0.36525700 & -1.22354300 \\ \mathrm{H} & -2.42334300 & -1.50990200 & 0.10935000 \\ \mathrm{H} & -3.02383500 & 0.13811900 & 0.29333900 \\ \mathrm{C} & -0.86323200 & -0.01930200 & 0.35967600 \\ \mathrm{H} & -0.83844800 & -0.13243400 & 1.44578100 \\ \mathrm{C} & 0.22408500 & -0.91756600 & -0.22689300 \\ \mathrm{H} & 0.00345500 & -1.95573700 & 0.02211700 \\ \mathrm{H} & 0.22643800 & -0.83050300 & -1.31400600 \\ \mathrm{C} & 1.61901600 & -0.57965200 & 0.28390400 \\ \mathrm{H} & 2.31855900 & -1.37793700 & -0.01019000 \\ \mathrm{H} & 1.64977700 & -0.52868200 & 1.37752000 \\ \mathrm{C} & -0.62933500 & 1.44855200 & 0.02106600 \\ \mathrm{H} & 0.31459400 & 1.81462300 & 0.41907800 \\ \mathrm{H} & -0.60792400 & 1.58615900 & -1.05907700 \\ \mathrm{H} & -1.42821600 & 2.06495100 & 0.42763500 \\ \mathrm{O} & 2.17321100 & 0.53936800 & -0.28408500\end{array}$


P2 Absolute energy: -272.373188 hartrees

$\begin{array}{lrrr}\mathrm{C} & -0.99865600 & 1.45744500 & 0.06213300 \\ \mathrm{H} & -1.34773400 & 1.54209100 & 1.09165000 \\ \mathrm{H} & -0.07913600 & 2.02997700 & -0.03194800 \\ \mathrm{H} & -1.74765700 & 1.91021000 & -0.58379000 \\ \mathrm{C} & -0.78251400 & -0.00611800 & -0.30050800 \\ \mathrm{H} & -0.39860200 & -0.06181600 & -1.31855900 \\ \mathrm{C} & 0.26502300 & -0.65907300 & 0.62269300 \\ \mathrm{H} & -0.09274100 & -0.60834600 & 1.65153500 \\ \mathrm{H} & 0.34223300 & -1.71544500 & 0.35658400 \\ \mathrm{C} & 1.60847400 & -0.04587500 & 0.55407300 \\ \mathrm{H} & 1.90160200 & 0.78255800 & 1.18072000 \\ \mathrm{C} & -2.09233100 & -0.77952700 & -0.22110700 \\ \mathrm{H} & -1.95059600 & -1.82370700 & -0.49144200 \\ \mathrm{H} & -2.48833800 & -0.74484500 & 0.79387400 \\ \mathrm{H} & -2.84001200 & -0.35329000 & -0.88638200 \\ \mathrm{O} & 2.20592500 & -0.13361800 & -0.67528700 \\ \mathrm{H} & 3.05360700 & 0.31044200 & -0.66365300\end{array}$

P3 Absolute energy: -272.365323 hartrees

$\begin{array}{llll}\text { C } & -2.17799300 & -0.66804300 & 0.23329100\end{array}$

$\mathrm{H} \quad-2.49975600 \quad-0.76408500 \quad-0.80273200$

$\mathrm{H} \quad-2.14268400 \quad-1.66310700 \quad 0.67080500$

$\mathrm{H} \quad-2.92542600 \quad-0.08524600 \quad 0.76581800$

$\begin{array}{llll}\text { C } & -0.81332400 & 0.00903900 & 0.29668800\end{array}$

$\mathrm{H} \quad-0.53345500 \quad 0.12905600 \quad 1.34740000$

C $\quad 0.22473600 \quad-0.81553000 \quad-0.38368200$

$\mathrm{H} \quad-0.06490300 \quad-1.72808100 \quad-0.88157000$

C $\quad 1.61653300 \quad-0.32736300 \quad-0.53904400$

$\mathrm{H} \quad 1.67404000 \quad 0.46826100 \quad-1.28515300$

$\mathrm{H} \quad 2.26816700 \quad-1.13259500 \quad-0.87879500$

$\begin{array}{llll}\mathrm{C} & -0.87658600 & 1.42069800 & -0.30369100\end{array}$

$\mathrm{H} \quad \begin{array}{llll}\mathrm{H} & 0.07770800 & 1.92924100 & -0.20124200\end{array}$

$\mathrm{H} \quad-1.13545900 \quad 1.36850500 \quad-1.36009000$

$\mathrm{H} \quad-1.63591100 \quad 2.01021800 \quad 0.20527600$

$\begin{array}{llll}\mathrm{O} & 2.13001700 & 0.26553300 & 0.65410800\end{array}$

$\mathrm{H} \quad 2.03734800 \quad-0.36923800 \quad 1.36605100$

P4 Absolute energy: -272.3708356 hartrees

$\begin{array}{llll}\text { C } & 1.67304400 & -1.21663500 & -0.02936300\end{array}$

$\mathrm{H} \quad 2.39494900 \quad-1.25125700 \quad 0.79639000$ 


$\begin{array}{lrrr}\mathrm{H} & 1.07833800 & -2.12553100 & 0.03183600 \\ \mathrm{H} & 2.25113600 & -1.23785700 & -0.95153900 \\ \mathrm{C} & 0.82031300 & -0.00026800 & 0.05244300 \\ \mathrm{C} & -0.35650400 & 0.02170300 & 0.97373500 \\ \mathrm{H} & -0.49558600 & -0.95668600 & 1.42999800 \\ \mathrm{H} & -0.19508400 & 0.74459300 & 1.78145700 \\ \mathrm{C} & -1.64925300 & 0.39935200 & 0.25374800 \\ \mathrm{H} & -1.56808900 & 1.40451100 & -0.16386800 \\ \mathrm{H} & -2.48395100 & 0.39038200 & 0.94763000 \\ \mathrm{C} & 1.39137300 & 1.29750400 & -0.40262900 \\ \mathrm{H} & 0.62717200 & 2.06393300 & -0.51006600 \\ \mathrm{H} & 2.12303100 & 1.67522800 & 0.32223600 \\ \mathrm{H} & 1.91341500 & 1.19605400 & -1.35232700 \\ \mathrm{O} & -1.96697200 & -0.53363900 & -0.76389400 \\ \mathrm{H} & -1.18338800 & -0.64420400 & -1.30819100\end{array}$

P5 Absolute energy: -272.3622605 hartrees

$\begin{array}{lccc}\mathrm{C} & 0.66676200 & 1.45634000 & -0.02670100 \\ \mathrm{H} & 0.50049700 & 1.71135900 & -1.06230300 \\ \mathrm{H} & 0.94260200 & 2.24960200 & 0.64811100 \\ \mathrm{C} & 0.87358200 & 0.03088300 & 0.34457900 \\ \mathrm{H} & 0.80666600 & -0.06734700 & 1.42936400 \\ \mathrm{C} & -0.16997400 & -0.89736900 & -0.28591600 \\ \mathrm{H} & -0.17163800 & -0.75904500 & -1.36817600 \\ \mathrm{H} & 0.11927100 & -1.93012400 & -0.08921900 \\ \mathrm{C} & -1.57837700 & -0.68330400 & 0.24302000 \\ \mathrm{H} & -1.57389500 & -0.75103700 & 1.33417400 \\ \mathrm{H} & -2.23772700 & -1.45947900 & -0.13402300 \\ \mathrm{C} & 2.28602100 & -0.41149600 & -0.08233300 \\ \mathrm{H} & 2.47068100 & -1.43731900 & 0.23009700 \\ \mathrm{H} & 2.37977000 & -0.35962000 & -1.16513200 \\ \mathrm{H} & 3.04651300 & 0.22829800 & 0.35660600 \\ \mathrm{O} & -2.15178000 & 0.54410200 & -0.17256600 \\ \mathrm{H} & -1.53658200 & 1.25157600 & 0.04514000\end{array}$

TS1 Absolute energy: -348.7783473 hartrees

$\begin{array}{lllr}\mathrm{C} & -2.16320000 & 1.29991900 & 0.07195300 \\ \mathrm{H} & -2.76787600 & 0.99987500 & 0.92749200 \\ \mathrm{H} & -1.68091200 & 2.24392000 & 0.31712400 \\ \mathrm{H} & -2.83243300 & 1.46605400 & -0.76907900 \\ \mathrm{C} & -1.13485200 & 0.22095300 & -0.25037300 \\ \mathrm{H} & -0.56295400 & 0.53383400 & -1.12360800\end{array}$




$\begin{array}{lrrr}\mathrm{C} & -0.16508900 & 0.07398400 & 0.91973800 \\ \mathrm{H} & 0.32554900 & 1.02876500 & 1.10726000 \\ \mathrm{H} & -0.72574200 & -0.18640700 & 1.82039700 \\ \mathrm{C} & 0.91265500 & -0.97814000 & 0.71626200 \\ \mathrm{H} & 0.46386900 & -1.97059200 & 0.65723900 \\ \mathrm{H} & 1.61426800 & -0.97492000 & 1.54882700 \\ \mathrm{C} & -1.82974200 & -1.09503900 & -0.58353300 \\ \mathrm{H} & -1.12804500 & -1.84077200 & -0.94726200 \\ \mathrm{H} & -2.32957000 & -1.49312500 & 0.30011500 \\ \mathrm{H} & -2.58310200 & -0.94472000 & -1.35342700 \\ \mathrm{O} & 1.60048700 & -0.82864900 & -0.50750200 \\ \mathrm{H} & 2.37137200 & -0.09968700 & -0.41497200 \\ \mathrm{O} & 2.64118500 & 1.14741300 & -0.27882300 \\ \mathrm{H} & 2.18355900 & 1.55760200 & -1.02378100\end{array}$

TS2 Absolute energy: -348.7817251 hartrees

$\begin{array}{lrrr}\mathrm{C} & 1.86726600 & 1.13581200 & -0.64234500 \\ \mathrm{H} & 1.92356900 & 0.80317700 & -1.67907600 \\ \mathrm{H} & 1.17532600 & 1.97168200 & -0.58611100 \\ \mathrm{H} & 2.85127900 & 1.49768400 & -0.35370200 \\ \mathrm{C} & 1.43666300 & -0.01510400 & 0.25984600 \\ \mathrm{H} & 1.29572400 & 0.37584800 & 1.26731200 \\ \mathrm{C} & 0.11299800 & -0.63187700 & -0.20139100 \\ \mathrm{H} & 0.24805400 & -1.08148800 & -1.18645300 \\ \mathrm{H} & -0.16675800 & -1.44136100 & 0.47605000 \\ \mathrm{C} & -1.03177700 & 0.35275100 & -0.28197500 \\ \mathrm{H} & -1.96614900 & -0.23967900 & -0.58176500 \\ \mathrm{H} & -0.89333600 & 1.10637300 & -1.05014900 \\ \mathrm{C} & 2.51208000 & -1.09502000 & 0.30098000 \\ \mathrm{H} & 2.21551000 & -1.92514900 & 0.93864500 \\ \mathrm{H} & 2.69008600 & -1.48663100 & -0.70011200 \\ \mathrm{H} & 3.45101500 & -0.69473300 & 0.67614200 \\ \mathrm{O} & -1.28767500 & 1.03318400 & 0.91894800 \\ \mathrm{H} & -1.31462000 & 0.39464400 & 1.63474200 \\ \mathrm{O} & -3.36754800 & -0.76151900 & -0.35891700 \\ \mathrm{H} & -3.65129900 & 0.06694300 & 0.05353500\end{array}$

TS3 Absolute energy: -348.7828297 hartrees

$\begin{array}{lrrr}\mathrm{C} & -1.89605000 & 1.24901400 & -0.08452200 \\ \mathrm{H} & -2.35605600 & 1.23503800 & 0.90356600 \\ \mathrm{H} & -1.30970500 & 2.15970000 & -0.17671500 \\ \mathrm{H} & -2.69227000 & 1.27172700 & -0.82471500\end{array}$




$\begin{array}{lrrr}\mathrm{C} & -1.01424400 & 0.02002100 & -0.27359500 \\ \mathrm{H} & -0.57386800 & 0.05370900 & -1.26845000 \\ \mathrm{C} & 0.11875200 & 0.04644100 & 0.73656600 \\ \mathrm{H} & 0.68646500 & 1.06039000 & 0.57997000 \\ \mathrm{H} & -0.25386000 & 0.09382600 & 1.76000500 \\ \mathrm{C} & 1.16454200 & -1.02845600 & 0.60142100 \\ \mathrm{H} & 0.72114700 & -2.01396300 & 0.75171900 \\ \mathrm{H} & 1.94169900 & -0.87994200 & 1.35012300 \\ \mathrm{C} & -1.83758600 & -1.26102100 & -0.14689600 \\ \mathrm{H} & -1.24851500 & -2.14530600 & -0.37563500 \\ \mathrm{H} & -2.23202700 & -1.36117400 & 0.86421900 \\ \mathrm{H} & -2.68047300 & -1.23486900 & -0.83365700 \\ \mathrm{O} & 1.71896300 & -0.93542900 & -0.71057800 \\ \mathrm{H} & 2.40486700 & -1.59420800 & -0.81705600 \\ \mathrm{O} & 1.58645600 & 1.92997300 & -0.07083800 \\ \mathrm{H} & 1.93676500 & 1.24272700 & -0.65988400\end{array}$

TS4 Absolute energy: -348.785609 hartrees

$\begin{array}{lrrr}\mathrm{C} & 2.20851500 & -0.10454200 & -0.65613900 \\ \mathrm{H} & 2.60420600 & -1.11999700 & -0.71163100 \\ \mathrm{H} & 2.13151200 & 0.28748100 & -1.66719300 \\ \mathrm{H} & 2.91801900 & 0.50617600 & -0.10439600 \\ \mathrm{C} & 0.85387300 & -0.11245900 & 0.02794700 \\ \mathrm{H} & 0.54288600 & 0.99885200 & 0.07811800 \\ \mathrm{C} & -0.17753400 & -0.83709300 & -0.82695900 \\ \mathrm{H} & -0.28640400 & -0.30867000 & -1.77334400 \\ \mathrm{H} & 0.20526300 & -1.83474600 & -1.05811600 \\ \mathrm{C} & -1.54341500 & -1.01067700 & -0.19970800 \\ \mathrm{H} & -1.47078400 & -1.59736300 & 0.71542900 \\ \mathrm{H} & -2.19728200 & -1.53684900 & -0.89421800 \\ \mathrm{C} & 0.93161200 & -0.61037700 & 1.45818600 \\ \mathrm{H} & 0.00203600 & -0.43856500 & 1.99365300 \\ \mathrm{H} & 1.14765400 & -1.68056200 & 1.47384900 \\ \mathrm{H} & 1.72668100 & -0.09862800 & 1.99366800 \\ \mathrm{O} & -2.08448200 & 0.27846100 & 0.09139400 \\ \mathrm{H} & -2.94508500 & 0.18398700 & 0.49889400 \\ \mathrm{O} & -0.04985100 & 2.31604900 & -0.01383600 \\ \mathrm{H} & -0.94235200 & 1.93369000 & 0.01486200\end{array}$

TS5 Absolute energy: -348.7819432 hartrees

$\begin{array}{llll}\mathrm{C} & -0.62568100 & 1.34148700 & 0.47665400 \\ \mathrm{H} & -0.74300100 & 1.24723100 & 1.55450900\end{array}$




$\begin{array}{lrrr}\mathrm{H} & 0.51245500 & 1.61014200 & 0.30694900 \\ \mathrm{H} & -1.14167800 & 2.22498200 & 0.11391100 \\ \mathrm{C} & -1.02003200 & 0.08740600 & -0.27689600 \\ \mathrm{H} & -0.61257600 & 0.15387200 & -1.28550000 \\ \mathrm{C} & -0.48032400 & -1.18040100 & 0.38525500 \\ \mathrm{H} & -0.95555500 & -1.30092600 & 1.36126500 \\ \mathrm{H} & -0.77535600 & -2.04619800 & -0.21181100 \\ \mathrm{C} & 1.02282000 & -1.20028600 & 0.58815500 \\ \mathrm{H} & 1.33359400 & -2.14732800 & 1.02742100 \\ \mathrm{H} & 1.33402100 & -0.40241100 & 1.25519800 \\ \mathrm{C} & -2.54325100 & -0.00021600 & -0.37918000 \\ \mathrm{H} & -2.84563600 & -0.90225600 & -0.90749000 \\ \mathrm{H} & -2.98728000 & -0.02662700 & 0.61508000 \\ \mathrm{H} & -2.94915300 & 0.85840600 & -0.90763000 \\ \mathrm{O} & 1.73831400 & -0.97043100 & -0.62607700 \\ \mathrm{H} & 1.47420800 & -1.61670600 & -1.28216600 \\ \mathrm{O} & 1.79897200 & 1.85402200 & -0.10741300 \\ \mathrm{H} & 1.93648400 & 0.99115000 & -0.53574300 \\ & --------------------------------------------------------------------------\end{array}$


Table S3. Conformational-vibrational rotational partition functions of the reactant (R) and of the transition states (TS1-TS5) of the five reactions by three methods: ${ }^{a}$

- MS-T: multistructural torsional anharmonicity approximation with coupled torsional-potential anharmonicity

- MS-LQH: multistructural local quasiharmonic approximation including all structures

- SS-QH: single-structure-quasiharmonic vibrational-rotational partition function

\begin{tabular}{|c|c|c|c|c|c|c|c|}
\hline & $T(\mathrm{~K})$ & $\mathrm{R}$ & TS1 & TS2 & TS3 & TS4 & TS5 \\
\hline \multirow[t]{7}{*}{$Q_{\text {con-rovib }}^{\mathrm{MS}-\mathrm{T}}$} & 298 & $7.82 \times 10^{-67}$ & $1.50 \times 10^{-68}$ & $3.04 \times 10^{-68}$ & $6.99 \times 10^{-69}$ & $9.21 \times 10^{-70}$ & $1.54 \times 10^{-69}$ \\
\hline & 400 & $1.04 \times 10^{-46}$ & $2.55 \times 10^{-47}$ & $5.46 \times 10^{-47}$ & $1.28 \times 10^{-47}$ & $1.74 \times 10^{-48}$ & $5.47 \times 10^{-48}$ \\
\hline & 600 & $3.83 \times 10^{-26}$ & $1.80 \times 10^{-25}$ & $4.08 \times 10^{-25}$ & $1.05 \times 10^{-25}$ & $1.85 \times 10^{-26}$ & $9.49 \times 10^{-26}$ \\
\hline & 800 & $5.78 \times 10^{-15}$ & $1.69 \times 10^{-13}$ & $4.02 \times 10^{-13}$ & $1.13 \times 10^{-13}$ & $2.45 \times 10^{-14}$ & $1.43 \times 10^{-13}$ \\
\hline & 1000 & $1.30 \times 10^{-7}$ & $1.42 \times 10^{-5}$ & $3.51 \times 10^{-5}$ & $1.06 \times 10^{-5}$ & $2.58 \times 10^{-6}$ & $1.61 \times 10^{-5}$ \\
\hline & 1500 & $3.40 \times 10^{4}$ & $3.50 \times 10^{7}$ & $9.12 \times 10^{7}$ & $3.12 \times 10^{7}$ & $8.40 \times 10^{6}$ & $5.79 \times 10^{7}$ \\
\hline & 2400 & $8.50 \times 10^{15}$ & $9.96 \times 10^{19}$ & $2.72 \times 10^{20}$ & $1.04 \times 10^{20}$ & $2.79 \times 10^{19}$ & $2.17 \times 10^{20}$ \\
\hline \multirow[t]{7}{*}{$Q_{\text {con-rovib }}^{\mathrm{MS}-\mathrm{LQH}}$} & 298 & $6.25 \times 10^{-67}$ & $1.54 \times 10^{-68}$ & $4.82 \times 10^{-68}$ & $8.54 \times 10^{-69}$ & $1.92 \times 10^{-69}$ & $3.92 \times 10^{-69}$ \\
\hline & 400 & $7.78 \times 10^{-47}$ & $2.34 \times 10^{-47}$ & $8.52 \times 10^{-47}$ & $1.63 \times 10^{-47}$ & $3.70 \times 10^{-48}$ & $1.61 \times 10^{-47}$ \\
\hline & 600 & $2.77 \times 10^{-26}$ & $1.47 \times 10^{-25}$ & $6.32 \times 10^{-25}$ & $1.51 \times 10^{-25}$ & $4.64 \times 10^{-26}$ & $3.39 \times 10^{-25}$ \\
\hline & 800 & $4.31 \times 10^{-15}$ & $1.36 \times 10^{-13}$ & $6.41 \times 10^{-13}$ & $1.89 \times 10^{-13}$ & $7.64 \times 10^{-14}$ & $6.08 \times 10^{-13}$ \\
\hline & 1000 & $1.04 \times 10^{-7}$ & $1.19 \times 10^{-5}$ & $5.95 \times 10^{-5}$ & $2.07 \times 10^{-5}$ & $1.00 \times 10^{-5}$ & $8.03 \times 10^{-5}$ \\
\hline & 1500 & $3.46 \times 10^{4}$ & $3.59 \times 10^{7}$ & $1.94 \times 10^{8}$ & $8.86 \times 10^{7}$ & $5.40 \times 10^{7}$ & $4.25 \times 10^{8}$ \\
\hline & 2400 & $1.38 \times 10^{16}$ & $1.67 \times 10^{20}$ & $9.34 \times 10^{20}$ & $5.46 \times 10^{20}$ & $3.87 \times 10^{20}$ & $2.99 \times 10^{21}$ \\
\hline \multirow[t]{7}{*}{$Q_{\mathrm{rovib}, 1}^{\mathrm{SS}-\mathrm{QH}}$} & 298 & $5.77 \times 10^{-68}$ & $2.43 \times 10^{-69}$ & $2.26 \times 10^{-69}$ & $6.36 \times 10^{-70}$ & $5.67 \times 10^{-70}$ & $2.26 \times 10^{-70}$ \\
\hline & 400 & $5.80 \times 10^{-48}$ & $2.57 \times 10^{-48}$ & $3.31 \times 10^{-48}$ & $8.16 \times 10^{-49}$ & $7.06 \times 10^{-49}$ & $2.51 \times 10^{-49}$ \\
\hline & 600 & $1.58 \times 10^{-27}$ & $1.06 \times 10^{-26}$ & $1.94 \times 10^{-26}$ & $4.18 \times 10^{-27}$ & $3.50 \times 10^{-27}$ & $1.12 \times 10^{-27}$ \\
\hline & 800 & $2.11 \times 10^{-16}$ & $7.76 \times 10^{-15}$ & $1.70 \times 10^{-14}$ & $3.45 \times 10^{-15}$ & $2.85 \times 10^{-15}$ & $8.66 \times 10^{-16}$ \\
\hline & 1000 & $4.59 \times 10^{-9}$ & $5.85 \times 10^{-7}$ & $1.44 \times 10^{-6}$ & $2.82 \times 10^{-7}$ & $2.30 \times 10^{-7}$ & $6.82 \times 10^{-8}$ \\
\hline & 1500 & $1.31 \times 10^{3}$ & $1.44 \times 10^{6}$ & $4.07 \times 10^{6}$ & $7.69 \times 10^{5}$ & $6.22 \times 10^{5}$ & $1.79 \times 10^{5}$ \\
\hline & 2400 & $4.63 \times 10^{14}$ & $5.67 \times 10^{18}$ & $1.75 \times 10^{19}$ & $3.25 \times 10^{18}$ & $2.61 \times 10^{18}$ & $7.41 \times 10^{17}$ \\
\hline
\end{tabular}

${ }^{a}$ The zero of energy for the partition functions is the energy of the lowest-energy classical equilibrium structure of the given species (reactant or transition state). 
Table S4. Ratios of MS-T rate constants to SS-QH rate constants ${ }^{\mathrm{a}}$

\begin{tabular}{llllll}
\hline $\mathrm{T}(\mathrm{K})$ & $\mathrm{R} 1$ & $\mathrm{R} 2$ & $\mathrm{R} 3$ & $\mathrm{R} 4$ & $\mathrm{R} 5$ \\
\hline 298 & 0.45 & 0.99 & 0.81 & 0.12 & 0.50 \\
400 & 0.55 & 0.92 & 0.88 & 0.14 & 1.21 \\
500 & 0.63 & 0.88 & 0.95 & 0.17 & 2.26 \\
600 & 0.70 & 0.87 & 1.04 & 0.22 & 3.50 \\
800 & 0.79 & 0.86 & 1.19 & 0.31 & 6.04 \\
1000 & 0.86 & 0.86 & 1.33 & 0.40 & 8.29 \\
1500 & 0.94 & 0.86 & 1.56 & 0.52 & 12.47 \\
2400 & 0.96 & 0.84 & 1.75 & 0.58 & 15.96 \\
\hline
\end{tabular}

${ }^{a}$ This ratio is defined as $\mathrm{F}_{\mathrm{act}}^{\mathrm{MS}-\mathrm{T}}$. 
Table S5. This table is the same as Table 5 except that it has more temperatures.

Computed standard-state entropy $(\mathrm{cal} / \mathrm{mol} / \mathrm{K})$, heat capacity $(\mathrm{cal} / \mathrm{mol} / \mathrm{K})$, and relative enthalpy $(\mathrm{kcal} / \mathrm{mol})^{a}$ for isopentanol using different methods

\begin{tabular}{|c|c|c|c|c|c|c|c|c|c|c|c|}
\hline \multirow[b]{2}{*}{$T(\mathrm{~K})$} & \multicolumn{4}{|c|}{$S_{T}^{0}$} & \multicolumn{4}{|c|}{$\overline{C_{P}^{0}(T)}$} & \multicolumn{3}{|c|}{$\boldsymbol{H}_{P}^{\mathbf{0}}(\boldsymbol{T})^{a}$} \\
\hline & MS-LQH & MS-T & $\mathrm{GA}^{b}$ & ${\mathrm{SS}-\mathrm{QH}^{c}}$ & MS-LQH & MS-T & $\mathrm{GA}^{b}$ & $\overline{\mathrm{SS}-\mathrm{QH}^{c}}$ & MS-LQH & MS-T & ${\mathrm{SS}-\mathrm{QH}^{\mathrm{c}}}$ \\
\hline 298 & 92.4 & 93.3 & 92.4 & 87.5 & 32.5 & 32.7 & 31.5 & 31.1 & 107.5 & 107.7 & 107.1 \\
\hline 300 & 92.6 & 93.5 & 92.6 & 87.7 & 32.7 & 32.8 & 31.7 & 31.3 & 107.6 & 107.8 & 107.2 \\
\hline 400 & 103.1 & 104.0 & 102.5 & 97.9 & 41.0 & 40.7 & 40.1 & 40.0 & 111.3 & 111.4 & 110.7 \\
\hline 500 & 113.1 & 113.9 & 111.6 & 107.7 & 48.7 & 47.9 & 47.3 & 48.0 & 115.8 & 115.9 & 115.1 \\
\hline 600 & 122.6 & 123.2 & 120.0 & 117.1 & 55.4 & 54.2 & 53.6 & 54.9 & 121.0 & 121.0 & 120.3 \\
\hline 700 & 131.6 & 132.0 & 127.4 & 126.0 & 61.1 & 59.5 & 58.9 & 60.7 & 126.8 & 126.7 & 126.1 \\
\hline 800 & 140.1 & 140.2 & 134.0 & 134.5 & 66.0 & 64.0 & 63.5 & 65.7 & 133.2 & 132.9 & 132.4 \\
\hline 900 & 148.1 & 148.0 & 140.0 & 142.4 & 70.2 & 67.9 & 67.5 & 69.9 & 140.0 & 139.5 & 139.2 \\
\hline 1000 & 155.7 & 155.3 & 144.4 & 150.0 & 73.9 & 71.3 & 70.9 & 73.6 & 147.2 & 146.4 & 146.4 \\
\hline
\end{tabular}

${ }^{a}$ The zero of energy is set to potential energy of the global minimum. ${ }^{b}$ This is calculated by using Benson's group additivity (GA) method. ${ }^{c}$ Calculated using the lowest-energy equilibrium conformer with its mirror image, but in the calculation of $F$ factors and in the SS-QH partition function listed in Table 3, the mirror image is correctly excluded. 
Table S6. This table is like Table 6 except with more significant figures. Ratios of MS-T product ratios to $\mathrm{SS}-\mathrm{QH}$ product ratios $^{a}$

\begin{tabular}{llllll}
\hline $\mathrm{T}(\mathrm{K})$ & $\mathrm{R} 1$ & $\mathrm{R} 2$ & $\mathrm{R} 3$ & $\mathrm{R} 4$ & $\mathrm{R} 5$ \\
\hline 298 & 0.46 & 1.00 & 0.82 & 0.12 & 0.51 \\
400 & 0.60 & 1.00 & 0.95 & 0.15 & 1.32 \\
600 & 0.80 & 1.00 & 1.19 & 0.25 & 4.02 \\
800 & 0.93 & 1.00 & 1.39 & 0.37 & 7.05 \\
1000 & 1.00 & 1.00 & 1.54 & 0.46 & 9.63 \\
1500 & 1.09 & 1.00 & 1.81 & 0.60 & 14.45 \\
2400 & 1.13 & 1.00 & 2.07 & 0.69 & 18.90 \\
\hline
\end{tabular}

${ }^{a}$ The R2 column is unity by definition. 
Table S7. Calculated rate constants using MP-CVT/SCT.

\begin{tabular}{cccccc}
\hline $\mathrm{T}(\mathrm{K})$ & $\mathrm{R} 1$ & $\mathrm{R} 2$ & $\mathrm{R} 3$ & $\mathrm{R} 4$ & $\mathrm{R} 5$ \\
\hline 298 & $1.00 \times 10^{-13}$ & $6.13 \times 10^{-12}$ & $3.18 \times 10^{-12}$ & $3.72 \times 10^{-12}$ & $3.61 \times 10^{-13}$ \\
400 & $9.54 \times 10^{-14}$ & $1.73 \times 10^{-12}$ & $9.85 \times 10^{-13}$ & $8.10 \times 10^{-13}$ & $2.29 \times 10^{-13}$ \\
500 & $1.29 \times 10^{-13}$ & $1.39 \times 10^{-12}$ & $7.41 \times 10^{-13}$ & $5.15 \times 10^{-13}$ & $3.07 \times 10^{-13}$ \\
600 & $1.77 \times 10^{-13}$ & $1.45 \times 10^{-12}$ & $7.26 \times 10^{-13}$ & $4.66 \times 10^{-13}$ & $4.43 \times 10^{-13}$ \\
700 & $2.40 \times 10^{-13}$ & $1.63 \times 10^{-12}$ & $7.88 \times 10^{-13}$ & $4.83 \times 10^{-13}$ & $6.26 \times 10^{-13}$ \\
800 & $3.18 \times 10^{-13}$ & $1.88 \times 10^{-12}$ & $8.94 \times 10^{-13}$ & $5.29 \times 10^{-13}$ & $8.57 \times 10^{-13}$ \\
900 & $4.11 \times 10^{-13}$ & $2.19 \times 10^{-12}$ & $1.03 \times 10^{-12}$ & $5.91 \times 10^{-13}$ & $1.14 \times 10^{-12}$ \\
1000 & $5.21 \times 10^{-13}$ & $2.55 \times 10^{-12}$ & $1.20 \times 10^{-12}$ & $6.67 \times 10^{-13}$ & $1.47 \times 10^{-12}$ \\
1500 & $1.35 \times 10^{-12}$ & $5.10 \times 10^{-12}$ & $2.45 \times 10^{-12}$ & $1.18 \times 10^{-12}$ & $4.05 \times 10^{-12}$ \\
1800 & $2.09 \times 10^{-12}$ & $7.21 \times 10^{-12}$ & $3.53 \times 10^{-12}$ & $1.59 \times 10^{-12}$ & $6.40 \times 10^{-12}$ \\
2000 & $2.70 \times 10^{-12}$ & $8.87 \times 10^{-12}$ & $4.38 \times 10^{-12}$ & $1.90 \times 10^{-12}$ & $8.33 \times 10^{-12}$ \\
2400 & $4.17 \times 10^{-12}$ & $1.28 \times 10^{-11}$ & $6.43 \times 10^{-12}$ & $2.61 \times 10^{-12}$ & $1.30 \times 10^{-11}$ \\
\hline
\end{tabular}


Table S8. This table is the same as Table 8 except that it has another temperature and more significant figures.

Comparisons of the rate constants for forward reactions by MS-CVT/SCT and SS-CVT /SCT. ${ }^{a}$

\begin{tabular}{ccccccccccc}
\hline & \multicolumn{2}{c}{$\mathrm{R} 1$} & \multicolumn{2}{c}{$\mathrm{R} 2$} & \multicolumn{2}{c}{$\mathrm{R} 3$} & \multicolumn{2}{c}{$\mathrm{R} 4$} & \multicolumn{2}{c}{$\mathrm{R} 5$} \\
\hline$T(\mathrm{~K})$ & $\mathrm{MS}$ & $\mathrm{SS}$ & $\mathrm{MS}$ & $\mathrm{SS}$ & $\mathrm{MS}$ & $\mathrm{SS}$ & $\mathrm{MS}$ & $\mathrm{SS}$ & $\mathrm{MS}$ & $\mathrm{SS}$ \\
\hline 298 & $8.77 \times 10^{-14}$ & $1.93 \times 10^{-13}$ & $2.86 \times 10^{-12}$ & $2.88 \times 10^{-12}$ & $1.50 \times 10^{-12}$ & $1.85 \times 10^{-12}$ & $2.51 \times 10^{-12}$ & $2.10 \times 10^{-11}$ & $1.09 \times 10^{-13}$ & $2.16 \times 10^{-13}$ \\
400 & $1.01 \times 10^{-13}$ & $1.83 \times 10^{-13}$ & $1.22 \times 10^{-12}$ & $1.32 \times 10^{-12}$ & $6.89 \times 10^{-13}$ & $7.86 \times 10^{-13}$ & $4.79 \times 10^{-13}$ & $3.47 \times 10^{-12}$ & $1.22 \times 10^{-13}$ & $1.00 \times 10^{-13}$ \\
500 & $1.40 \times 10^{-13}$ & $2.21 \times 10^{-13}$ & $1.14 \times 10^{-12}$ & $1.29 \times 10^{-12}$ & $5.70 \times 10^{-13}$ & $5.97 \times 10^{-13}$ & $2.81 \times 10^{-13}$ & $1.62 \times 10^{-12}$ & $1.83 \times 10^{-13}$ & $8.09 \times 10^{-14}$ \\
600 & $3.49 \times 10^{-13}$ & $4.40 \times 10^{-13}$ & $1.73 \times 10^{-12}$ & $2.02 \times 10^{-12}$ & $7.06 \times 10^{-13}$ & $5.92 \times 10^{-13}$ & $2.69 \times 10^{-13}$ & $8.57 \times 10^{-13}$ & $5.31 \times 10^{-13}$ & $8.79 \times 10^{-14}$ \\
800 & $3.49 \times 10^{-13}$ & $4.40 \times 10^{-13}$ & $1.73 \times 10^{-12}$ & $2.02 \times 10^{-12}$ & $7.06 \times 10^{-13}$ & $5.92 \times 10^{-13}$ & $2.69 \times 10^{-13}$ & $8.57 \times 10^{-13}$ & $5.31 \times 10^{-13}$ & $8.79 \times 10^{-14}$ \\
1000 & $5.75 \times 10^{-13}$ & $6.70 \times 10^{-13}$ & $2.42 \times 10^{-12}$ & $2.81 \times 10^{-12}$ & $9.40 \times 10^{-13}$ & $7.08 \times 10^{-13}$ & $3.33 \times 10^{-13}$ & $8.42 \times 10^{-13}$ & $9.04 \times 10^{-13}$ & $1.09 \times 10^{-13}$ \\
1500 & $1.51 \times 10^{-12}$ & $1.61 \times 10^{-12}$ & $4.98 \times 10^{-12}$ & $5.77 \times 10^{-12}$ & $1.87 \times 10^{-12}$ & $1.20 \times 10^{-12}$ & $5.72 \times 10^{-13}$ & $1.10 \times 10^{-12}$ & $2.42 \times 10^{-12}$ & $1.94 \times 10^{-13}$ \\
2400 & $4.75 \times 10^{-12}$ & $4.96 \times 10^{-12}$ & $1.27 \times 10^{-11}$ & $1.50 \times 10^{-11}$ & $4.74 \times 10^{-12}$ & $2.71 \times 10^{-12}$ & $1.21 \times 10^{-12}$ & $2.08 \times 10^{-12}$ & $7.47 \times 10^{-12}$ & $4.68 \times 10^{-13}$ \\
\hline
\end{tabular}

${ }^{a}$ The MS-CVT/SCT results are labeled MS; the SS-CVT/SCT ones are labeled SS. 


\section{Torsional anharmonicity factors}

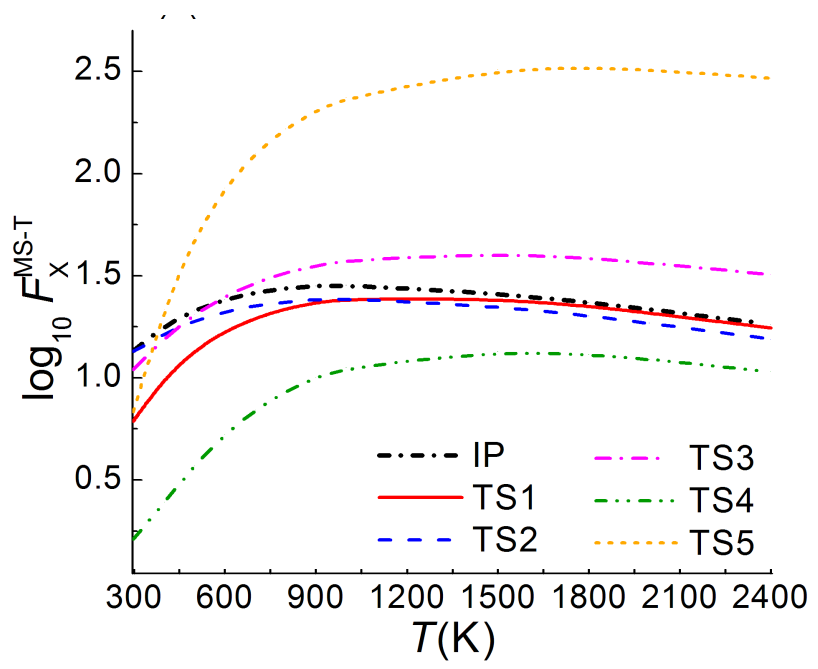

Figure S1. Multistructural torsional anharmonicity factors for each species $X(X=\mathrm{R}, \mathrm{TS} 1, \mathrm{TS} 2$, TS3, TS4, TS5). This plot illustrates the factors in the top half of Figure 3a as defined in Section 2.5. 


\section{Local periodicities}

All the local periodicities of structures of the reactant and of transition states TS1-TS5 are given for illustration in Figure S2. The physical meaning of the $M_{j, \tau}$ parameter is explained in detail in the original MS-T paper, ${ }^{1}$ but a rough explanation is that it tells us the average effective numbers of minima along the torsional coordinates. Figure S2 shows that the local periodicities are usually between 1.6 and 3.6. The figure shows clearly that the local periodicities for the reactant and transition states have different ranges. The most significant reason why we see a variety of $M_{j, \tau}$ values is the influence of hydrogen bonding in some of the structures.

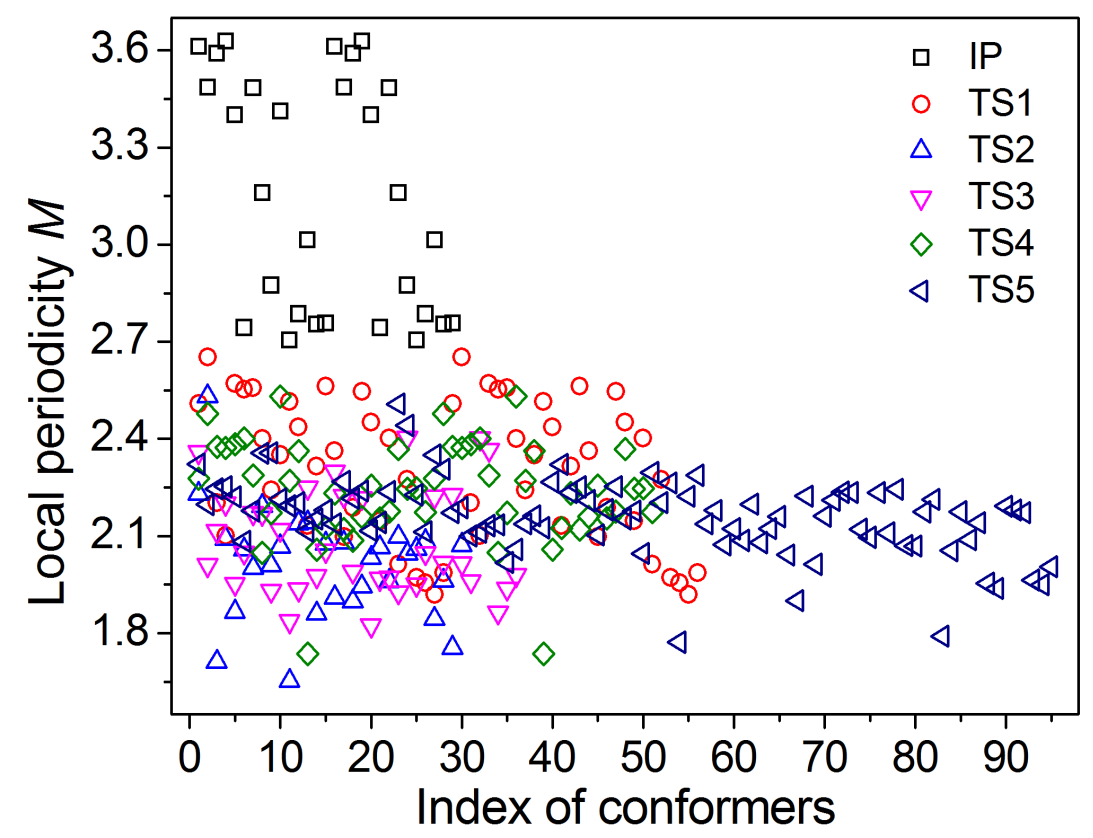

Figure S2. The unitless local periodicities $\mathrm{M}_{\mathrm{j}, \tau}$ for isopentanol (IP), TS1, TS2, TS3, TS4, and TS5. 


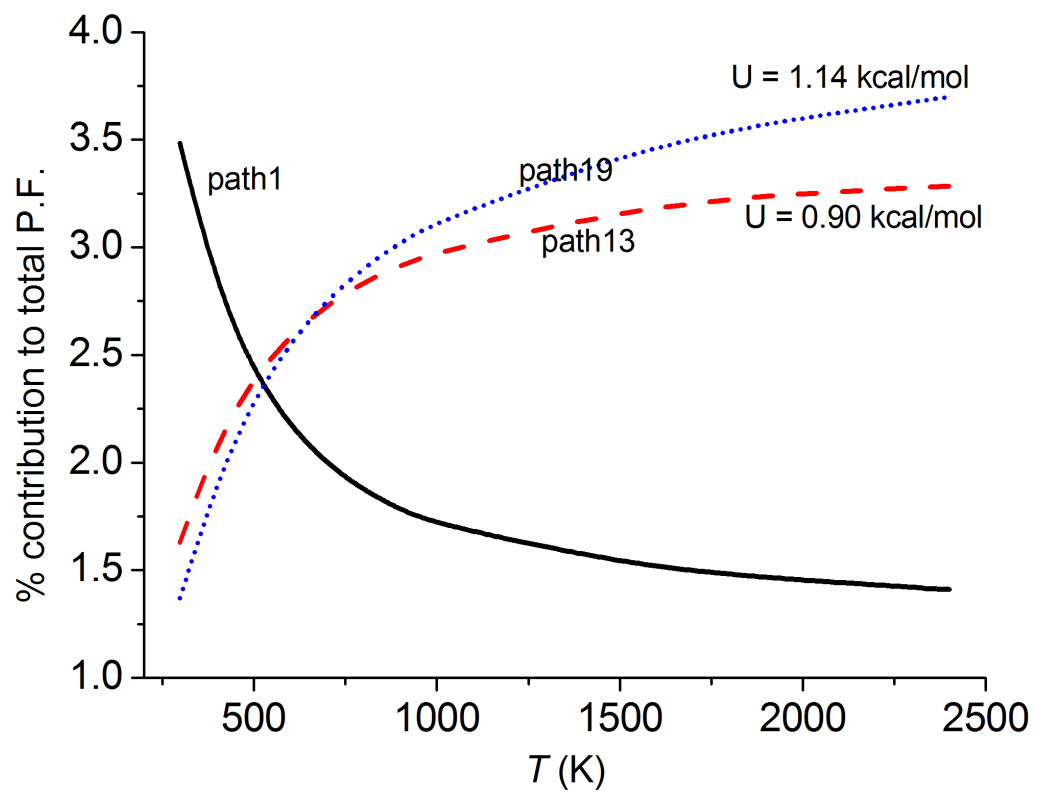

Figure S3. Percentage contribution of the lowest-energy and some high-energy transition structures to the total MS-T partition functions of transition states for R2. The black line is for the lowest-energy structures; blue and red lines represent ones of the high-energy structures. The energy labels are relative to the zero point-exclusive lowest-energy transition structure in the corresponding reaction. 


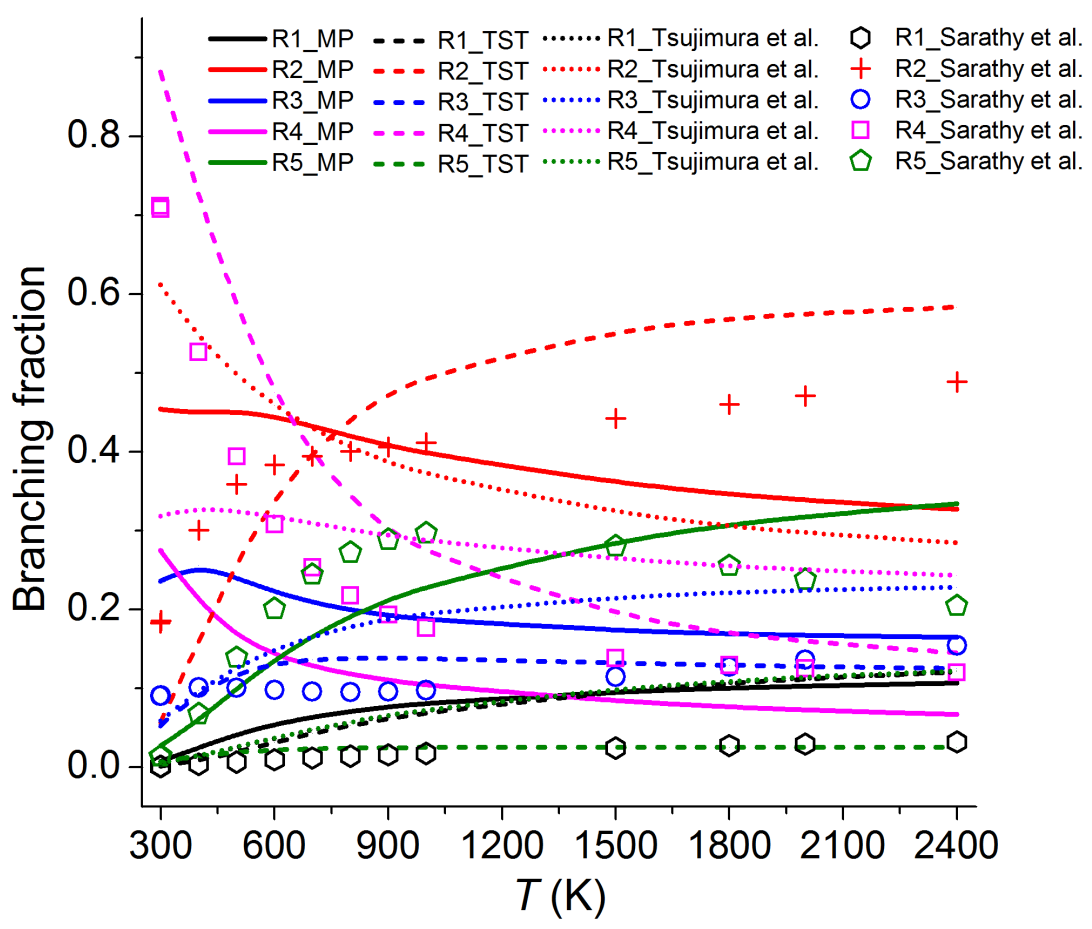

Figure S4. Branching fractions of R1-R5 as functions of temperature computed by MP-CVT/SCT (solid lines), by single-structural harmonic conventional TST (dashed lines), from Sarathy et al. ${ }^{9}$ (dotted lines), from Tsujimura et al. ${ }^{6}$ (symbols). The branching fraction of reaction $\mathrm{R} i$ is computed as the ratio of rate constant of $\mathrm{R} i$ to the overall rate constant (sum of the rate constants for all reactions). 


\section{Reference}

${ }^{1}$ Zheng, J.; Yu, T.; Papajak, E.; Alecu, I. M.; Mielke, S. L.; Truhlar, D. G. Practical methods for including torsional anharmonicity in thermochemical calculations on complex molecules: The internalcoordinate multi-structural approximation. Phys. Chem. Chem. Phys. 2011, 13, 10885-10907. 